\title{
Theme: Immunization
}

Intranasal and sublingual delivery of Inactivated Polio G Vaccine (Vaccine. 2017 Apr 8. pii: S0264-410X(17)304425. doi: 10.1016/j.vaccine.2017.03.090. [Epub ahead of print $]$ )

The current study evaluated the possibilities of polio vaccination via mucosal surfaces using Inactivated polio vaccine (IPV) based on attenuated Sabin strains. Mice received three immunizations with trivalent SIPV via intramuscular injection, or via the intranasal or sublingual route. The need of an adjuvant for the mucosal routes was investigated as well, by testing SIPV in combination with the mucosal adjuvant cholera toxin.

Both intranasal and sublingual sIPV immunization induced systemic polio-specific serum IgG in mice that were functional as measured by poliovirus neutralization. Intranasal administration of sIPV plus adjuvant induced significant higher systemic poliovirus type 3 neutralizing antibody titers than sIPV delivered via the intramuscular route. Moreover, mucosal sIPV delivery elicited polio-specific IgA titers at different mucosal sites (IgA in saliva, fecal extracts and intestinal tissue) and IgA-producing Bcells in the spleen, where conventional intramuscular vaccination was unable to do so.

Comment: This study seems to be a polio strategist's delight wherein the best attributes of the two main polio vaccines are utilized in a single product. Mucosal responses are important because they prevent establishment of initial infection at the port of entry and subsequent dissemination to other sites. If the results are reproduced in human studies, the intranasal and sublingual routes may prove to be valuable approaches for routine vaccination and outbreak control in the post-eradication era.

\section{Tdap administered during pregnancy is effective in protecting infants against pertussis (Pediatrics. 2017;139:e20164091).}

In this retrospective cohort study of infants born at Kaiser Permanente Northern California from 2010 to 2015, the researchers estimated the effectiveness of maternal pertussis vaccination for protecting newborns against pertussis in the first 2 months of life and in the first year of life accounting for each infant DTaP dose. Among 148,981 newborns, the vaccine effectiveness of maternal tetanus toxoid, reduced diphtheria toxoid, acellular pertussis (Tdap) vaccine was 91.4\% (95\% CI 19.5 to 99.1$)$ during the first 2 months of life and $69.0 \%$ (95\% CI 43.6 to 82.9 ) during the entire first year of life. The vaccine effectiveness was $87.9 \%$ (95\% CI 41.4 to 97.5$)$ before infants had any DTaP vaccine doses, $81.4 \%$ (95\% CI 42.5 to 94.0 ) between doses 1 and 2, 6.4\% (95\% CI " 165.1 to 66.9) between doses 2 and 3 , and $65.9 \%$ (95\% CI 4.5 to 87.8$)$ after infants had $3 \mathrm{DTaP}$ doses.

Comment: Maternal vaccination with Tdap currently seems to be the most effective strategy to protect young infants against pertussis. All other strategies including 'cocoon vaccination' with Tdap have not been effective, as it relies on herd immunity, which is not sufficiently well induced by acellular pertussis (aP)containing products. In fact, this is the only valid and effective indication of use of available Tdap vaccine now, considering unexpectedly faster waning of aP vaccine induced immunity. However, there are certain issues like optimum timing of the maternal Tdap vaccination, possible interference with first infant dose of pertussis-containing vaccine, and hyper-immunization with tetanus and diphtheria toxoid; these need to be sorted out.

Interchangebility of pneumococcal conjugate vaccines (PCVs) - Divergent memory B-Cell responses in a mixed infant PCV schedule (Pediatr Infect Dis J. 2017;36:e130-5)

Vaccine-induced immunity against pneumococcal infection relies on the generation of high concentrations of antibody and B cell memory. Both the 10- and the 13-valent pneumococcal conjugate vaccines (PCV-10 and PCV-13) effectively reduce disease caused by vaccine serotypes. It is unknown whether the generation of $B$ cell memory requires several doses of the same vaccine or whether different $\mathrm{PCV}$ s are interchangeable.

Children in the United Kingdom $(n=178)$ who had previously received PCV-13 at 2 and 4 months were randomized $1: 1$ to receive a PCV-13 or PCV-10 booster at age 12 months. Peripheral blood memory B cells $\left(\mathrm{B}_{\mathrm{MEM}}\right)$ were quantified before and at 1 and 12 months after vaccination using a cultured enzymelinked immunospot assay for pneumococcal serotypes 1, 3, 4, 9V, 14, 19A, and diphtheria and tetanus toxoid. Correlations between $\mathrm{B}_{\mathrm{MEM}}$ frequencies and simultaneously measured antibody (IgG and opsonophagocytic assay) was also assessed.

A significant rise in post-booster $\mathrm{B}_{\mathrm{MEM}}$ frequency was seen for 5 out of 6 serotypes in the PCV-13 group and none in the PCV-10 group. In the PCV-13 group, there was a particularly large increase in serotype 3-specific $\mathrm{B}_{\mathrm{MEM}}$ associated with only a small increase in antibody. Post-booster $B_{\text {MEM }}$ responses correlated positively with antibody, but correlations between pre-booster $\mathrm{B}_{\mathrm{MEM}}$ and subsequent $\mathrm{B}_{\mathrm{MEM}}$ and antibody responses were inconsistent.

Comments: The scarcity of the vaccines - such as recently witnessed with IPV - would bring the issue of interchangebility of PCVs in the limelight. The above study suggests that immunizing with a PCV-containing polysaccharides conjugated to a novel carrier protein is not sufficient to generate a rapid and strong B-cell memory response, at least when primary vaccination with PCV-13 is followed by a booster dose of PCV-10.

VIPIN M VAShishtha vipinipsita@gmail.com 